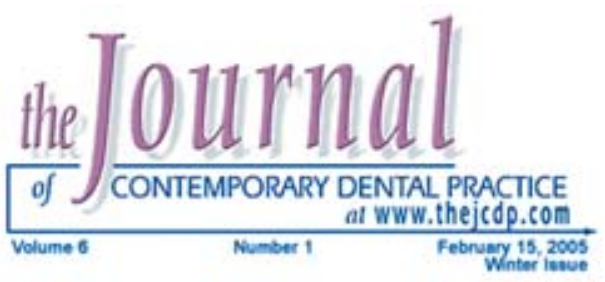

\title{
Correlation of Different MRI Characteristics of Anterior Disc Displacement With Reduction and Without Reduction
}

\author{
Sevgi Şener, DDS, PhD; Faruk Akgünlü, DDS, PhD
}

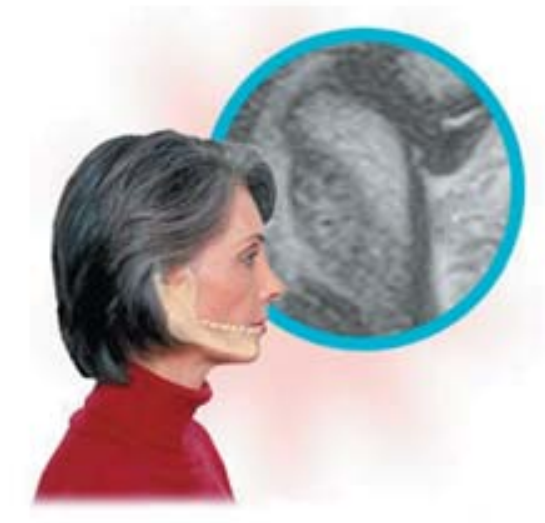

Abstract

The aim of this study was to evaluate the correlations between magnetic resonance imaging (MRI) findings in anterior displacement with reduction (ADDR) and anterior displacement without reduction (ADDWR). Two hundred patients with temporomandibular disorder (TMD) symptoms were examined clinically by a clinician experienced with this disorder. There were 130 joints with ADDR and 45 joints with ADDWR; 88 patients were identified according to the clinical and MRI diagnoses of both observers. The MRI characteristics such as position, signal intensity, morphology of disc, degenerative changes, effusion, scar tissue, avascular necrosis, and condylar hypermobility were evaluated in the individual with ADDR and ADDWR. The Chi-square test was used to determine the correlation between MRI characteristics in the ADDR and the ADDWR. There were significant relationships between degenerative changes and increased signal intensity, degenerative changes and side-way displacement, effusion and the deformation of the disc, effusion and subluxation, increased signal intensity and subluxation, elongation deformation and increased signal intensity, and elongation deformation and side-way displacement in ADDR. There were significant relationships between degenerative changes and effusion, degenerative changes and decreased signal intensity, scar tissue and avascular necrosis, deformation of disc and increased signal intensity, as well as deformation of disc and decreased signal intensity in ADDWR. These MRI findings also correlated with the clinical findings, however, this correlation appears to be a synergistic pattern rather than a set of clear-cut relationships. The

(C) Seer Publishing 
correlations between these MRIs and different clinical findings such as pain and dysfunction also must be evaluated. Existing correlations must be considered in the diagnosis and treatment planning of TMD, and signs and symptoms of TMD should be monitored after treatment.

Keywords: MRI, TMJ, internal derangement, disc displacement

Citation: Şener S, Akgünlü F. Correlation of Different MRI Characteristics of Anterior Disc Displacement With Reduction and Without Reduction. J Contemp Dent Pract 2005 February;(6)1:026-036.

\section{Introduction}

Magnetic resonance imaging (MRI) of the temporomandibular joint (TMJ) can provide information on the position and structure of the disc, the quantitiy of synovial fluid, condition of the bone, posterior attachment and retrodiscal tissues, the bone marrow, periarticular tissues, and adhesions. ${ }^{1}$ These MRI characteristics are important considerations in the treatment planning of temporomandibular disorder (TMD) and can relate to the pain and disorder of patients. ${ }^{2}$

The issue of structural characteristics in the diagnosis and assessment of TMD is important because many clinicians base their diagnosis, etiological hypothesis, and treatment regimens almost entirely on an assesment of structural characteristics of a particular patient. Most prior research has been conducted as simple pairwise tests of TMJ MRI, but this assumes an "all or none" role and ignores the simultaneous contribution of multiple factors which inevitably occur in biologic systems. ${ }^{2}$ MRI findings correlate with each other and with clinical findings. Generally the MRI studies of the TMJ have been concerned with the relationship between clinical findings and MRI characteristics. ${ }^{3}$
The aim of this study was to evaluate the correlations between MRI findings in anterior displacement with reduction (ADDR) and anterior displacement without reduction (ADDWR).

\section{Method and Materials}

Two hundred patients with TMD symptoms were examined clinically by an exprienced clinician for their TMD according to Okeson. ${ }^{8-9}$ MRIs were obtained for these patients and were interpreted by the same experienced clinician and radiologist who were blind to each other.

One hundred thirty (130) patients with ADDR and 45 patients with ADDWR having the same $\mathrm{MRI}$ and clinical diagnosis were included in the study. Clinical examination and MRI interpretation were also assessed as blind to each other. Images were obtained by a 1.5 Tesla MR device (United Medical Technologies Corp, Ft. Myers, FL, USA) and $6.8 \mathrm{~cm}$ surface coil. T1, $\mathrm{T} 2$, and proton density images were obtained on the sagittal and coronal planes with a $3 \mathrm{~mm}$ slice thickness and $0.5 \mathrm{~mm}$ slice gap. Table 1 shows the MRI parameters used in this study.

The diagnosis of ADDWR and ADDR were made on MRIs according to Larheim. ${ }^{10}$ In the ADDR the posterior band of the disc was in front of the superior portion of the condylar head on closed mouth position, but the disc was located between

Table 1. MRI parameters used in present study.

\begin{tabular}{|c|c|c|c|c|}
\hline & TRJT & Matrix & FOV & NEX \\
\hline $\begin{array}{c}\text { T1 } \\
\text { Images }\end{array}$ & $500 / 16$ & $192^{\star} 256$ & $11 \mathrm{~cm}$ & 1 \\
\hline $\begin{array}{l}\text { Proton } \\
\text { Density }\end{array}$ & $2009 / 16$ & $192^{\star} 256$ & $11 \mathrm{~cm}$ & 1 \\
\hline T2 imges & $2009 / 80$ & $192^{\star} 256$ & $11 \mathrm{~cm}$ & 1 \\
\hline
\end{tabular}


the articular eminence and the superior portion of condylar head in the open mouth position. In the ADDWR the posterior band of the disc was in front of the superior part of the condylar head both in closed and open mouth positions. The following were noted on the MRIs:

- Side-way displacements (in addition to anterior displacement, not pure side-way displacement)

- Degenerative changes

- Effusion

- Signal intensity of articular disc

- Morphology of articular disc

- Hypermobility of condyle

- Avascular necrosis

- Scar tissue

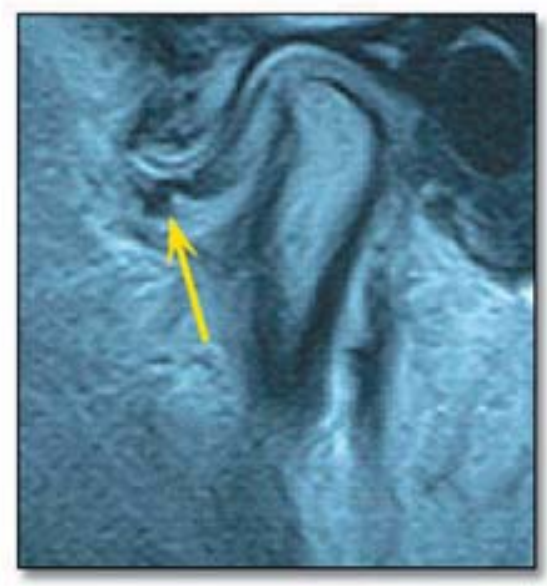

Figure 1. Degenerative change and side-way displacement (lateral) of a case with ADDR.

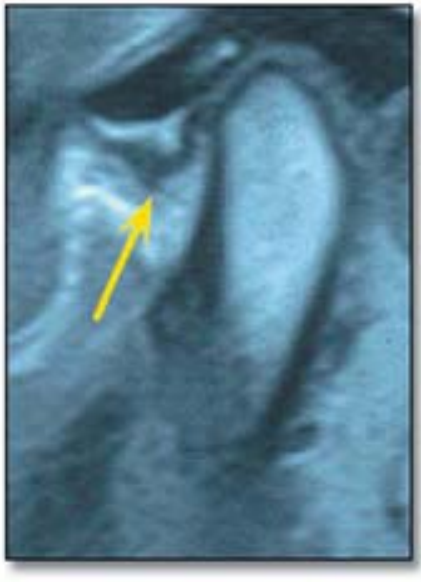

Figure 2. Effusion and degenerative change of a case with ADDWR.

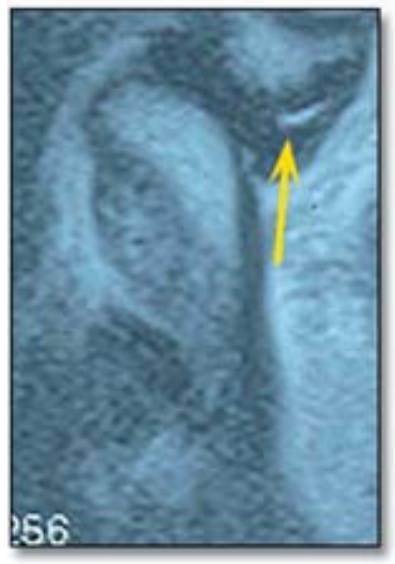

Figure 3. Subluxation and effusion of a case with ADDR.
Side-way displacements in addition to anterior displacement were classified into lateral and medial displacements. Lateral and medial displacements were identified as a bulging of the articular disc laterally or medially on coronal images according to Katzberg and Westesson ${ }^{11}$ (Figure 1).

Erosion, flattening, and osteophites of the articular surfaces are referred to as degenerative changes on coronal images according to Larheim and Katzberg and Westesson. ${ }^{10-11}$ (Figures 1 and 2) The level or severity of degenerative changes was ignored.

The hyperintense areas on T2 weighted images are described as effusion according to Adame et al., Rudisch et al., and Schellas and Wilkes., 12, 13 (Figures 2 and 3 ) The level or severity of effusion was ignored.

Biconcave, (bow-tie configurations) of the disc were accepted as normal disc morphology in accordance with Helms et al. and Dijkgraaf et al. ${ }^{14-15}$ Morphologic changes are classified into: elongation (thinning) (Figure 4), thickening of posterior band (TPB) (Figure 5) and flexion (folding) (Figure 6) and full deformation (destruction of disc completely) in this study.

The intermediate-low signal intensities were accepted as normal for the articular disc according to Helms et al. ${ }^{14}$ The changes in the signal intensity were classified into increased signal intensity (Figure 4-5) or decreased signal intensity (Figure 6).

If the head of the condyle was in front of the articular eminence on maximum opening of the mouth, then hypermobility was diagnosed in that joint according to Gynther et al., Shorey and Campbell, and Faucart et al. ${ }^{16,17,18}$ (Figure 3)

Low MRI signal intensity in the bone marrow of the condyle was accepted as avascular necrosis, and areas of low signal intensity that extended into the joint space irregulary were accepted as adhesions or scar tissues as seen in the coronal and sagittal images according to Katzberg. ${ }^{11}$ (Figure 7) 


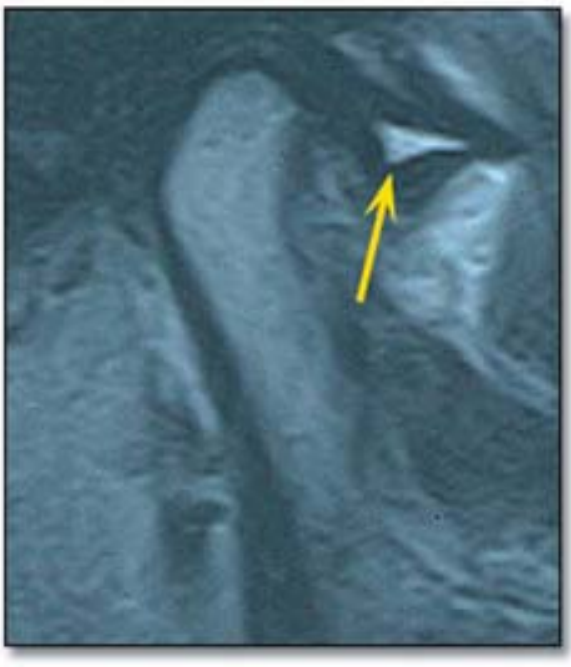

Figure 4. Elongation deformation and increased signal intensity of posterior band of a case with ADDWR.

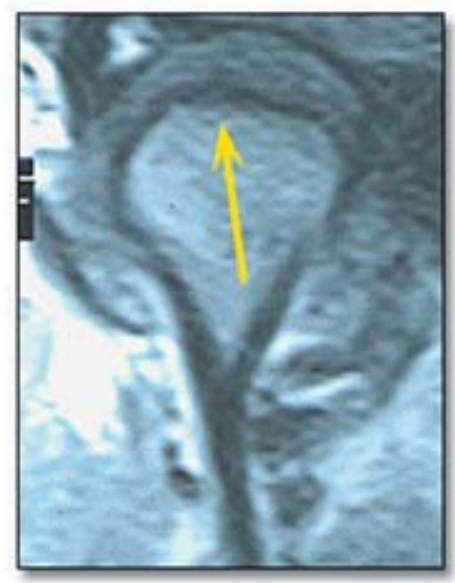

Figure 6. Flexion deformity and decreased signal intensity of a case with ADDWR.

MRI investigations were made on a negatoscope and blind to clinical examinations. Datums were scored as present (1) and absent (0). The Chisquare test was used to investigate the correlation of MRI findings between ADDR and ADDWR.

\section{Results}

The Chi-square test showed there were significant relationships between degenerative changes and increased signal intensity $(p<0.05)$ as well as side-way displacement $(p<0.05)$. It also showed significant relationships between effusion and the deformation of the disc $(p<0.05)$ and subluxation $(p<0.05)$. Significant relationships were also found between increased signal intensity and subluxation $(p<0.05)$, elongation deformation and

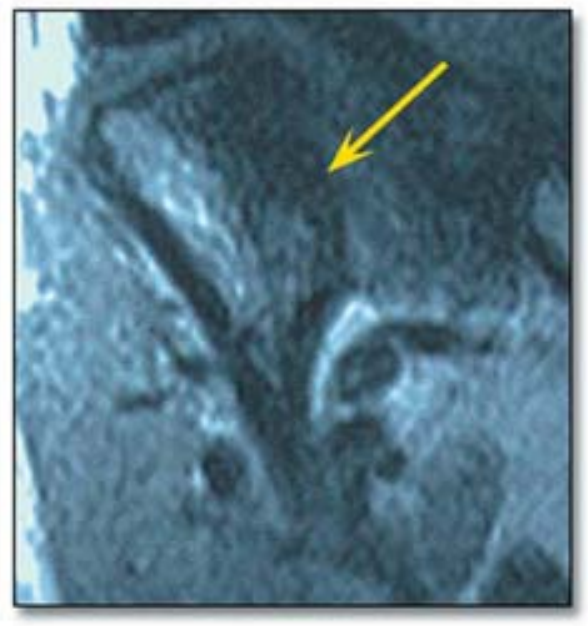

Figure 5. Increased signal intensity, multiple scar tissues, and thickening of posterior band of a case with ADDWR.

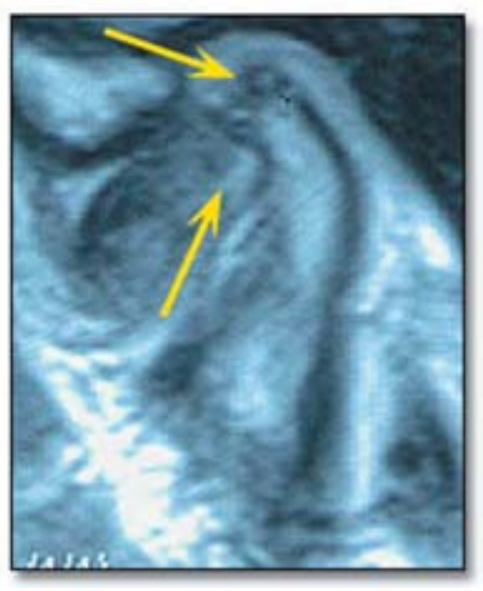

Figure 7. Scar tissue and avascular necrosis.

increased signal intensity $(p<0.001)$, as well as elongation deformation and side-way displacement $(p<0.05)$ in ADDR.

Furthermore, the Chi-square test showed there were significant relationships between degenerative changes and effusion $(p<0.05)$, degenerative changes and decreased signal intensity $(p<0.05)$, scar tissue and avascular necrosis $(p<0.05)$, deformation of disc and increased signal intensity $(p<0.05)$, deformation of disc and decreased signal intensity $(p<0.05)$ in ADDWR. The frequencies of MRI parameters are presented in Tables 2-7. Tables 8 and 9 show the correlation between MRI characteristics in ADDR and ADDWR. 
Table 2. The frequency of degenerative changes and effusion in ADDR and ADDWR.

\begin{tabular}{|c|c|c|}
\hline & $\begin{array}{c}\text { Degenerative } \\
\text { Changes }\end{array}$ & Efiusion \\
\hline ADDR & $84.6 \%$ & $34.6 \%$ \\
\hline ADDWR & $84.4 \%$ & $48.8 \%$ \\
\hline TOTAL & $84.5 \%$ & $38.2 \%$ \\
\hline
\end{tabular}

Table 3. The frequency of disc deformation in ADDR and ADDWR.

\begin{tabular}{|c|c|c|c|c|}
\hline & Disc Deformation (Total) & Flexion & TPB & Elongation \\
\hline ADDR & $28.4 \%$ & $3.8 \%$ & $28.4 \%$ & $20 \%$ \\
\hline ADDWR & $57.7 \%$ & $24.4 \%$ & $57.7 \%$ & $31.1 \%$ \\
\hline
\end{tabular}

Table 4. The frequency of the double and triple deformation types in ADDR and ADDWR.

\begin{tabular}{|c|c|c|c|}
\hline & $\begin{array}{c}\text { Flexion and } \\
\text { elongation }\end{array}$ & TPB and elongation & $\begin{array}{c}\text { TPB and } \\
\text { elongation and } \\
\text { flexion }\end{array}$ \\
\hline ADDR & $0.76 \%$ & $2.3 \%$ & $0.76 \%$ \\
\hline ADDWR & $6.6 \%$ & $2.2 \%$ & $2.2 \%$ \\
\hline
\end{tabular}

Table 5. The frequency of increased signal intensity, decreased signal ingensity, and total signal intensity changes (SIC) in ADDR and ADDWR.

\begin{tabular}{|c|c|c|c|}
\hline & IS & DS & SIC \\
\hline ADOR & $11.5 \%$ & $0.76 \%$ & $12.3 \%$ \\
\hline ADOWR & $28.8 \%$ & $68.8 \%$ & $97.7 \%$ \\
\hline TOTAL & $20.7 \%$ & $23.7 \%$ & $44.4 \%$ \\
\hline
\end{tabular}

Table 6. The frequency of medial, lateral, and total side-way displacement in ADDR and ADDWR.

\begin{tabular}{|c|c|c|c|}
\hline & W & MD & SD (total) \\
\hline ADOR & $1.5 \%$ & $35.3 \%$ & $36.9 \%$ \\
\hline ADDWR & $2.2 \%$ & $66.6 \%$ & $68.8 \%$ \\
\hline TOTAL & $2.2 \%$ & $56.2 \%$ & $58.5 \%$ \\
\hline
\end{tabular}

Table 7. The frequency of scar tissues, avascular necrosis, and hypermobility in ADDR and ADDWR.

\begin{tabular}{|c|c|c|c|}
\hline & Scar tissues & AN & Hypermobility \\
\hline ADOR & $35 \%$ & 0 & $31.5 \%$ \\
\hline ADOWR & $64.4 \%$ & $6.6 \%$ & 0 \\
\hline TOTAL & $42.8 \%$ & $2.2 \%$ & $30.3 \%$ \\
\hline
\end{tabular}

Table 8. Correlations between MRI charecteristics in ADDR.

\begin{tabular}{|c|c|c|c|c|}
\hline & Degerative Changes & Efiusion & Increased signal & Elongation \\
\hline Increased signal & 0.05 & - & - & 0.001 \\
\hline Side-way Displacement & 0.05 & - & - & 0.05 \\
\hline Disk deformation & - & 0.05 & - & - \\
\hline Subluxcation & - & 0.05 & 0.05 & - \\
\hline
\end{tabular}

Table 9. Correlations between MRI charecteristics in ADDWR.

\begin{tabular}{|c|c|c|c|c|}
\hline & Effusion & Decreased Signal & Avaskular necrosis & Increased signall \\
\hline Disc deformation & $\cdot$ & 0.05 & $\cdot$ & 0.05 \\
\hline Scar Tissue & $\cdot$ & $\cdot$ & 0.05 & $\cdot$ \\
\hline Degerative Changes & 0.05 & 0.05 & $\cdot$ & $\cdot$ \\
\hline
\end{tabular}




\section{Discussion}

Yilmaz and Toller stated there was a significant correlation between disc deformity and condylar degeneration and there was no significant relationship between the disc deformities and articular effusion. ${ }^{19}$ Segami et al. stated there was no correlation between either adhesion or degenerative changes and adhesion and effusion. ${ }^{20}$ In this study there was a significant relationship between condylar flattening and disc deformation in ADDR as well as between degenerative changes and elongation of the disc in ADDWR. The correlation between degenerative changes and deformation of discs may indicate the disc becomes displaced and deformed and does not employ effectively. Therefore, development of degenerative changes will be facilitated because the disc does not locate uniformly and homogenously between articular surfaces. Furthermore, it may be considered degenerative changes may result in discal deformation. Further longitudinal studies are needed to address this issue.

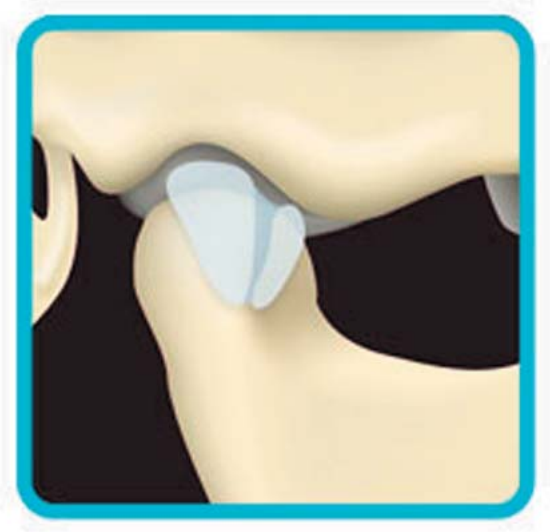

Normal Closed Disc Placement

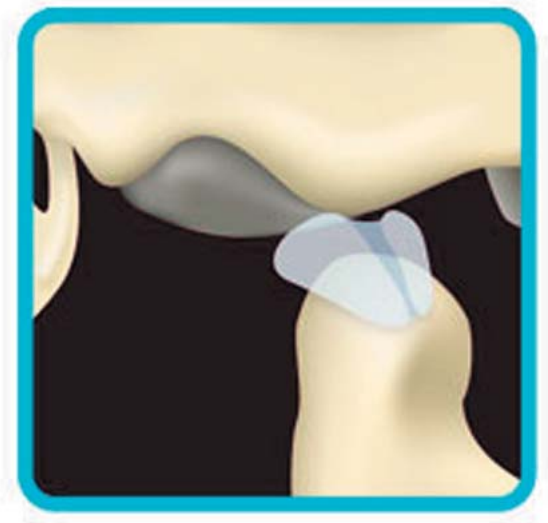

Normal Open Disc Placement
There was a significant relationship between the disc deformity and articular effusion in ADDR, but such a correlation was not found in ADDWR although the percentage of effusion and discal deformation was higher in ADDWR than ADDR in the present study. These findings suggest the development of the effusion effect from various MRIs, clinical findings, and clinical conditions. Furthermore, internal derangement type appeared not to directly affect the development of effusion.

Adame et al. stated effusion was not relevant to avascular necrosis but associated it with osteophytes. ${ }^{5}$ Osteophytes are proliferative degenerative changes of articular surfaces. They appear as little spicules on the articular surfaces, especially on the anterior portion of the condyle. In this study effusion was not relevant to avascular necrosis in both disc displacement types but rather degenerative changes associated with effusion in ADDWR. These findings suggest avascular necrosis and osteoarthiritic changes are separate entities in accordance with Sano et al. ${ }^{21}$ Because the percentage of effusion and degenerative changes were higher in ADDWR in this study, effusion and degenerative changes may be correlated with each other. Helms et al. stated decreased signal intensity was associated with osteoarthrosis. ${ }^{14}$ In this study osteoarthrosis was associated with increased signal intensity in ADDR and with decreased signal intensity in ADDWR. These findings suggest the following:

- The proportion of increased signal intensity was higher in ADDR and decreased signal intensity was higher in ADDWR.

- Osteoarthrosis can be present in both disc displacement types.

- If there is a real relationship between osteoarthrosis and signal intensity changes, discs give different responses to degenerative changes in every patient.

- It is possible signal intensity changes are associated with the type of the osteoarthrosis (proliferative changes such as osteophytes or degenerative changes such as flattening and erosion). 
Faucart et al. ${ }^{18}$ stated rotational displacement may be considered as an advanced stage in disc condyle dislocation. In the present study there was a significant relationship between side-way displacement and osteoarthrosis. If osteoarthrosis is considered as an advanced stage of internal derangement, this relationship may be explained. It may be inferred as the condyle becomes more flat, the disc displaces more easly in the coronal plane, especially medially.

Adame et al. ${ }^{5}$ stated effusion was related to degenerative changes. The findings of our study are in accordance with their findings in ADDWR but not in ADDR cases. This finding may suggest the following:

- The severity of degenerative changes may be greater in ADDWR cases.

- Because the percentage of disc deformity was higher in ADDWR, the cases with effusion were higher than ADDR cases.

- Various factors synergistically facilitate the development of effıısion

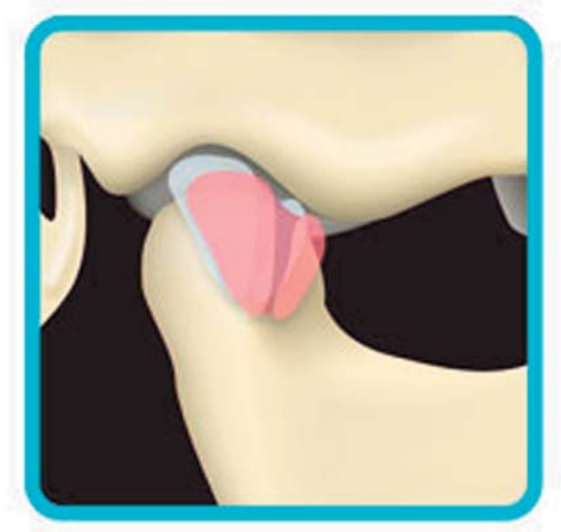

ADDR (closed)

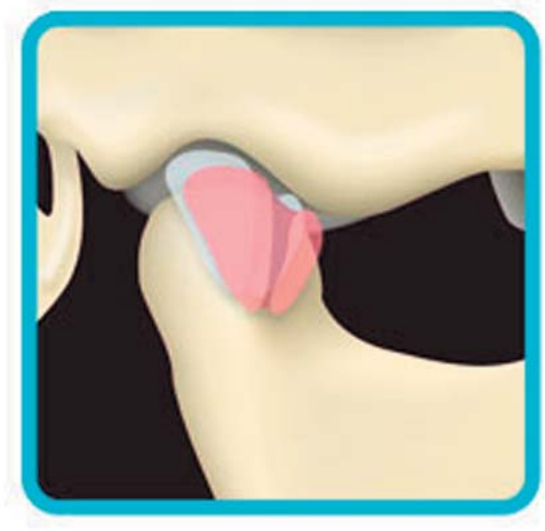

ADDWR (closed)
There was a significant relationship between scar tissue and development of avascular necrosis in this study. Furthermore, the prevalance of these types of changes are significantly greater in the cases with ADDWR than cases with ADDR. These findings reflect adhesions and avascular necrosis can result from long-term overloading of the TMJ.

Although the proportion of medial displacement and osteoarthrosis was higher in ADDWR, there was a siginificant relationship between medial displacement and osteoarthrosis in ADDR. Although this relationship was siginificant statistically $(p<0.05)$, it could not be explained.

The proportion of the subluxation was $30 \%$ in ADDR. There was a significant relationship between:

- Subluxation and effusion

- Subluxation and increased signal intensity in ADDR

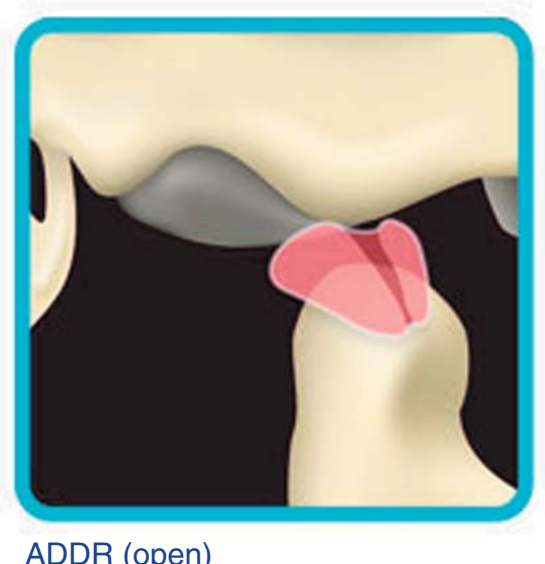

ADDR (open)

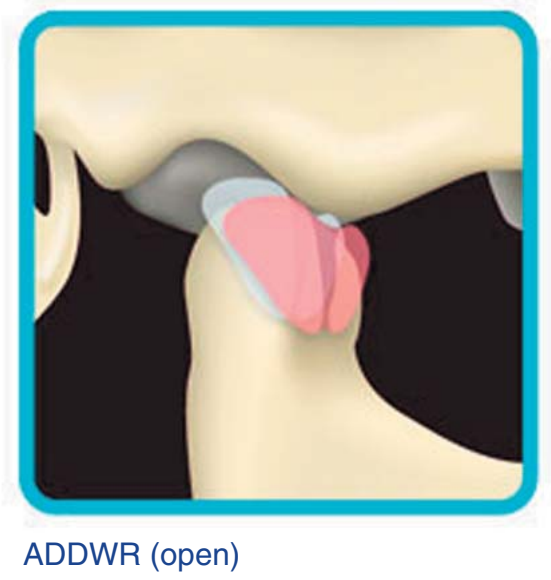


The relationship between subluxation, generalized joint laxity, and internal derangement needs to be investigated etiologically. These findings also suggest the subluxation of the condyle may cause inflamation and the presence of effusion also may reflect this inflamation.

The most significant relationship was between elongation deformation of the disc and increased signal intensity in ADDR in the present study $(p<0.001)$. Although there were cases with increased signal intensity in the ADDWR, the proportion of increased signal intensity was higher in ADDR than in ADDWR. Furthermore, if the increased signal indicates discal edema, then the elongation of the disc may be the most prevalent cause of discal edema.

In this study there was a significant relationship between:

- Disc deformation and increased signal intensity in ADDR

- Disc deformation and decreased signal intensity in ADDWR

These findings suggest different signal intensities can develop from the same disc deformation type and discs can respond differently to the same type of deformation. It can also be stated pathologic changes on the disc can result from different factors working synergistically. In addition to these issues the following must also be considered:

- The proportion of decreased signal intensity was more in ADDR

- The proportion of increased signal intensity was more in ADDWR

- Most of the cases in the present study with ADDWR had been in the acute phase in this study

\section{Conclusion}

$M R I$ is a non-invasive method to evaluate the configuration, position, and signal intensity of the TMJ disc, degenerative changes in the joint, and the bone marrow with excellent soft tissue resolution. These MRI findings correlate with each other and with clinical findings. However, the correlation between MRI findings appear to be as a synergistic pattern rather than as clear-cut relationships. The MRI findings such as osteroarthiritic changes and effusion can be found in both disc displacement types, but keep in mind the severity of these conditions can be different. While the MRI findings such as signal intensity changes, deformations of disc, scar tissues, avascular necrosis, and side-way displacement are found most frequently in ADDWR, the prevalance of the subluxation is higher in ADDR. These findings suggest as the dysfunction progress disc becomes more deformed, signal intensity alters and scar tissue and avascular necrosis develop. Side-way displacement, in addition to anterior displacement (rotational displacement), is a more complicated condition. The cause-result relationship must be investigated between anterior and side-way displacement.

It is important the correlation between MRI and various clinical findings such as pain and dysfunction be evaluated and all existing correlations considered in the diagnosis and treatment planning of TMD. This is also true with regard to the signs and symptoms experienced by patients 
after treatment.

References

1. Milano V, Desitate A, Bellino R, et al. Magnetic resonance imaging of temporomandibular disorders: classification, prevalance and interpretation of disc displacement and deformation. Dentomaxillofac Radiol. 2000 29: 352-361.

2. Emshoff R, Brandilmaier I, Bertram S, et al. Relative odds of temporomandibular joint pain as a function of magnetic resonance imagingfindings of internal derangement, osteoarthrosis, effusion, and bone marrow edema. Oral Surg Oral Med Oral Pathol Oral Radiol Endod. 2003; 95: 437-44.

3. Cholitgul W, Nishiyama H, Sasai T, et al. Clinical and magnetic resonance imaging findings in temporomandibular disc displacement. Dentomaxillofac Radiol. 1997; 26: 183-188.

4. Emshoff R, Puffer P, Rudisch A, et al. Temporomandibular joint pain: relationship to internal derangement type, osteoarthrosis and synovial fluid mediator level of tumor necrosis factor-alfa. Oral Surg Oral Med Oral Pathol Oral Radiol Endod. 2000; 90: 442-9.

5. Adame GC, Monje F, Munoz M, et al. Effusion in magnetic resonance imaging of the temporomandibular joint: A study of 123 joints. J Oral Maxillofac Surg. 1998; 56: 314-318.

6. Takahashi T, Nagai Hseki H, Fukuda M. Relationship between joint effusion, joint pain and protein levels in jont lavage fluid of patient with internal derangement and osteoarthirtis of the temporomandibular joint. J Oral Maxillofac Surg. 1999; 57: 1187-1193.

7. Emshoff R, Rudisch A. Validity of diagnostic criteria for temporomandibular disorders. Oral Surg Oral Med Oral Pathol Oral Radiol Endod. 2001; 91: 50-5.

8. Okeson JP. Management of temporomandibular disorders and occlusion. Mosby; Chapter 2; 246-61.

9. Okeson JP. Current teminology and diagnostic classification scheme., Oral Surg Oral Med Oral Pathol Oral Radiol Endod. 1997, 83: 61-64.

10. Larheim TA. Current trends in temporomandibular joint imaging, Oral Surg Oral Med Oral Pathol Oral Radiol Endod. 1995; 80: 555-576.

11. Katzberg RW, Westesson PL. Temporomandibular joint. WB Saunders Comp, Philedelphia,1993, pp 208-9.

12. Rudisch A, Innerhorfer K, Bertram S, et al. Magnetic resonance imaging findings of internal derangement and effusion in patient with unilateral temporomandibular joint pain. Oral Surg Oral Med Oral Pathol Oral Radio Endod. 2001; 92: 566-71

13. Schellas KP, Wilkes $\mathrm{CH}$. Temporomandibular joint inflammation: comparison of MR fast scanning with T1- and T2- weighted imaging tecniques. AJR Am J Roentgenol. 1989; 153: 93-8

14. Helms CA, Kaban LB, Mc Neil C. Temporomandibular joint: Morphology and signal intensity characteristics of the disk at MR imaging. Radiology 1989; 172: 817-820.

15. Dijkgraaf LC, De Bont LGM, Boering G. Three dimensional visualization of the temporomandibular joint: a computerized multisectional autopsy study of disk position and configuration. Dentomaxillofac Radiol. 2000; 29: 352-61.

16. Gynether GW, Tronje G, Holmlund AB. Radiographic changes in the temporomandibular joint patients with generalized osteoarthiritis and rheumatoid arthiritis Oral Surg Oral Med Oral Pathol Oral Radiol Endod. 1996; 81: 613-618.

17. Shorey CW, Campbell JH. Dislocation of the temporomandibular joint. Oral Surg Oral Med Oral Pathol Oral Radiol Endod. 2000; 89: 662-8.

18. Faucart JM, Carpentier P, Pajoni D, et al. MR of 732 TMJs: anterior, rotational, partial and sideways displacements. Eur J Radiol. 1998; 28: 86-94.

19. Yilmaz N, Toller M. Magnetic resonance imaging evaluation of temporomandibular joint disc deformities in relation to type of disc displacement, J Oral Maxillofac Surg. 2001; 58: 860-5.

20. Segami N, Nishimura M, Kaneyama K, et al. Does joint effusion on T2 magnetic resonance images reflect synovitis? Comparison of arthroskopic findings in internal derangements of the temporomandibular joint. Oral Surg Oral Med Oral Pathol Oral Radiol Endod. 2001; 92: 341-5.

21. Sano T, Westesson PL, Larheim TA, et al. Osteoarthiritis and abnormal bone marrow of the mandibular condyle. Oral Surg Oral Med Oral Pathol Oral Radiol Endod. 1999; 87: 243-52. 

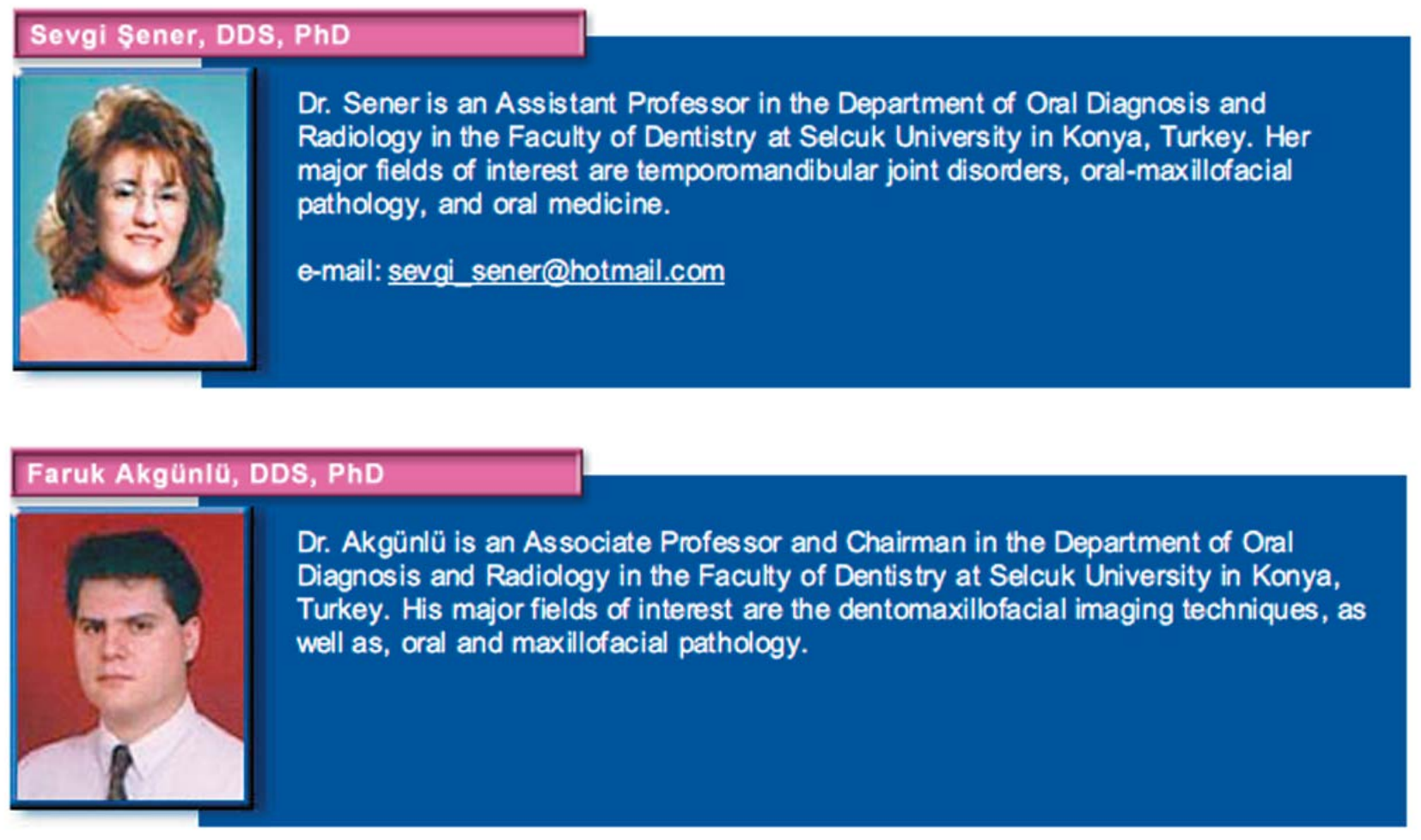